\title{
Quantity-Based Dispatching Models for Collection Centers in Reverse Supply Chains
}

\author{
Mehmet Alegoz and Onur Kaya \\ Anadolu University Department of Industrial Engineering, 26555 Eskisehir Turkey \\ mehmetalegoz@anadolu.edu.tr,onur_kaya@anadolu.edu.tr
}

\begin{abstract}
In this study, we focus on a collection center which collects an end-of-life (EOL) product, disassembles it, checks the reusability of its components and puts the reusable components in a warehouse until next dispatch to the manufacturer. Arrival times, batch sizes of arrivals and quality of components are assumed to be random. In this setting, we aim to determine the optimal dispatching quantity so as to maximize the profit of the collection center. For this problem, we mainly focus on two cases. In the first case, the acquisition fee is fixed, i.e. it is a parameter of the problem, and in the second case, it is assumed to be a variable effecting the rate of arrival, i.e. if the acquisition fee is high, arrival rate is also high and vice versa. For both cases, we develop renewal-reward process based dispatching models. Our first model determines the optimal quantity only, while our second model determines optimal quantity and optimal acquisition fee in a coordinated manner. We conclude the study by providing numerical examples and sensitivity analysis.
\end{abstract}

Keywords: Closed-Loop Supply Chains, Collection, Renewal-Reward Process, Dispatching

\section{Introduction}

Closed-loop supply chains focus on taking back products from customers and recovering added value by reusing the entire product, and/or some of its modules, components, and parts. Over the past years, closed-loop supply chains have gained considerable attention in industry and academia [1]. Effective, efficient and robust supply chains provide a sustainable competitive advantage for countries and firms, and help them to cope with increasing environmental turbulences and more intense competitive pressures [2]. Initially, the growing attention on reverse logistics and closed-loop supply chain issues originated with public awareness [3]. Then governmental legislation forced producers to take care of their end-of-life (EOL) products. For instance, the Waste Electrical and Electronic Equipment (WEEE) directive became European law in 2003, which contains mandatory requirements on collection, recycling, and recovery for all types of electrical goods, with a minimum rate of 4 kilograms per head of population per annum [3].

There are various papers in the literature which focus on different aspects of the collection process such as evaluating collection channel alternatives [4-7], sorting issue in collection [8-10], optimal order quantities and times [11-12]. In this study, we focus on dispatching and acquisition fee decisions of collection centers and propose quantity based dispatching models. In quantity based dispatching policy, a critical quantity is determined and the dispatching is made every time the amount of inventory exceeds that quantity. Because of its ease of use, quantity based dispatching approaches are one of the most preferred policies in real life.

The rest of the paper is organized as follows. In second part, we present the problem in detail. In third part, we introduce our models. Computational results and sensitivity analysis are given in fourth and fifth parts respectively. Finally, we conclude the study in part six. 


\section{Problem Definition}

We focus on a collection center that collects an EOL product composed of two components, component 1 and component 2, and sells the reusable components to a remanufacturer. EOL products are brought to the collection center by end users in batches and the collection center pays them an acquisition fee per product. Arrival times and batch sizes are assumed to be random. Upon arrival, each product is disassembled and basic quality control is made with a fixed quality control cost. By this way, reusability of component 1 and component 2 are determined. After this control, reusable components are sent to the warehouse of the collection center to be stored until the next dispatch and others are sent to landfill. In each dispatch, the collection center obtains a revenue by selling the reusable components to the manufacturer. Each component has a different probability of reusability and brings a different revenue. The system is illustrated below in Fig. 1.

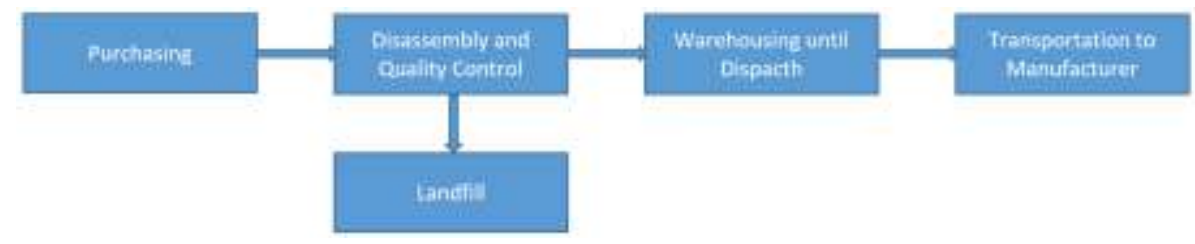

Fig. 1: The System

In this system, the collection center faces with four types of costs. These are acquisition fee (purchasing cost of one unit of EOL product), quality control cost, holding cost (as a result of holding the reusable components in warehouse) and transportation cost (as a result of transporting the reusable components from warehouse of collection center to manufacturer).

In this study, we focus on the quantity based dispatching heuristics such that dispatching will be made when the amount of EOL products brought to the collection center reaches to a certain level of $Q$. The problem is determining the optimal value of $Q$ to maximize the profit of the company. We propose renewal-reward theory based models for this problem. The reader may refer to [13] for detailed explanation of renewal-reward process and applications of it to the manufacturer's dispatching problem.

\section{Model}

In this part of study, we introduce the models but before that, we present the parameters and decision variables of the model as in Table 1.

TABLE I: Parameters and Decision Variables

\begin{tabular}{cl}
\hline \hline$S_{n}$ & Time of $n^{\text {th }}$ arrival \\
$X_{n}$ & Interarrival times (time between $S_{n-1}$ and $\left.S_{n}\right)$ \\
$D_{n}$ & Total inventory in warehouse after $n^{\text {th }}$ arrival \\
$Y_{n}$ & Batch size of $n^{\text {th }}$ arrival \\
$\lambda$ & Rate of first poisson process (process of arrivals) \\
$\alpha$ & Rate of second poisson process (process of batches) \\
$p_{1}$ & Probability of having a reusable Component 1 from an EOL product. \\
$p_{2}$ & Probability of having a reusable Component 2 from an EOL product. \\
$r_{1}$ & Revenue obtained by selling one unit of Component 1 \\
$r_{2}$ & Revenue obtained by selling one unit of Component 2 \\
$c_{p}$ & Unit purchasing cost of EOL product \\
$c_{q}$ & Unit quality control cost of EOL product \\
$h_{1}$ & Holding cost per one unit of Component 1 per unit time \\
$h_{2}$ & Holding cost per one unit of Component 2 per unit time \\
$\mathrm{K}$ & Fixed transportation cost \\
$\delta$ & Scale economy parameter $(0<\delta<1)$ \\
$N(Q)$ & Number of arrivals up to quantity $Q$ \\
\hline \hline
\end{tabular}


Moreover, let us define two poisson processes to use in our models. First poisson process is the process of arrivals. In this process, $X_{n}$ values are exponentially distributed with rate $\lambda$. Second possion process is the process of batches. Similarly, in this process $Y_{n}$ values are Poisson distributed with parameter $1 / \alpha$. We define $N(Q)$ (the number of arrivals up to quantity $Q$ ) as $\max \left(n: D_{n} \leq Q\right)$. After these definitions, we are finally ready to introduce the models.

\subsection{Fixed Acquisition Fee}

In this subsection, we assume that acquisition fee is fixed. In other words, it is a parameter of the problem. As it is mentioned before, EOL products arrive in batches. Letting $n=N(Q)+1$, total holding times of batches can be written as follows, where $T=\sum_{j=1}^{N(Q)+1} X_{j}$ denotes the dispatching time of the products.

$$
Y_{1}\left(T-S_{1}\right)+Y_{2}\left(T-S_{2}\right)+Y_{3}\left(T-S_{3}\right)+\cdots+Y_{n}\left(T-S_{n}\right)
$$

By this context, expected total holding time will be as in Equation (2).

$$
\begin{gathered}
E\left[\sum_{i=1}^{N(Q)+1} \sum_{j=1}^{N(Q)+1} Y_{i} X_{j}-\sum_{i=1}^{N(Q)+1} Y_{i} S_{i}\right]=E\left[E\left[\sum_{i=1}^{n} \sum_{j=1}^{n} Y_{i} X_{j}-\sum_{i=1}^{n} Y_{i} \sum_{j=1}^{i} X_{j}\right] \mid N(Q)+1=n\right] \\
=E[Y] E[X] E^{2}[N(Q)+1]-E[Y] E[X] E[(N(Q)+1)(N(Q)+2) / 2] \\
=E[Y] E[X]\left(E^{2}[N(Q)+1]-\frac{E\left[N(Q)^{2}+3 N(Q)+2\right]}{2}\right)
\end{gathered}
$$

The variables $Y$ (batch size) and $X$ (interarrival times) are independent from each other and the above equations are obtained by conditioning on $N(Q)$. Since interarrival times are independent and exponentially distributed, $S$ (arrival time) is written as sum of interarrival times in the first equation. Note that, since interarrival times are exponentially distributed and batch quantities are Poisson distributed, $\mathrm{E}[\mathrm{X}]=1 / \lambda$ and $\mathrm{E}[\mathrm{Y}]=1 / \alpha$. In addition, $\mathrm{E}[N(Q)]=\alpha Q$ and Variance $[N(Q)]=\alpha Q$. Then, $E\left[N(Q)^{2}\right]=\operatorname{Variance}[N(Q)]+$ $(E[N(Q)])^{2}=\alpha Q+\alpha^{2} Q^{2}$. By using these facts and some basic algebraic relations, expected total holding time will be obtained as in Equation (3).

$$
\frac{E[Y] E[X]\left[2 \alpha^{2} Q^{2}+4 \alpha Q+2-\left(\alpha Q+\alpha^{2} Q^{2}+3 \alpha Q+2\right)\right]}{2}=\frac{E[Y] \alpha^{2} Q^{2}}{2 \lambda}=\frac{\alpha Q^{2}}{2 \lambda}
$$

By using the obtained expected total holding time, it is possible to calculate expected long run average holding cost. Note that probabilities of obtaining reusable component 1 and component 2 are $p_{1}$ and $p_{2}$ respectively. By this context, expected long run average holding cost can be obtained as in Equation (4).

$$
\frac{E[\text { holding cost }]}{T}=\frac{\left(p_{1} h_{1}+p_{2} h_{2}\right)\left[\frac{\alpha Q^{2}}{2 \lambda}\right]}{\frac{[\alpha Q+1]}{\lambda}}=\frac{\left(p_{1} h_{1}+p_{2} h_{2}\right) \alpha Q^{2}}{2 \alpha Q+2}
$$

The collection center obtains a revenue for each component they sell to the manufacturer. Therefore, expected long run average revenue will be as follows.

$$
\frac{E[\text { revenue }]}{T}=\left(r_{1} p_{1}+r_{2} p_{2}\right) E[Y] \lambda
$$

The collection center pays an acquisition fee and has a quality control cost for each returned EOL product. Expected long run average purchasing and quality control cost will be as in Equation (6).

$$
\frac{E[\text { purchasing and quality control cost }]}{T}=\left(c_{p}+c_{q}\right) E[Y] \lambda
$$

Components are sent to manufacturer via owned vehicle. For this reason, the collection center has a transportation cost. This cost includes a fixed and a variable element. Fixed element includes costs which are independent from the amount transported such as the wage of driver, Variable element includes costs which are dependent to the amount transported such as cost of fuel oil. We can write expected long run average transportation cost as follows. 


$$
\frac{E[\text { transp. cost }]}{T}=\frac{K \lambda}{\alpha Q+1}+\frac{\left[\left(p_{1}+p_{2}\right) E[Y](\alpha Q+1)\right]^{\delta}}{\frac{[\alpha Q+1]}{\lambda}}=\frac{K \lambda}{\alpha Q+1}+\lambda\left[\left(p_{1}+p_{2}\right) E[Y]\right]^{\delta}[\alpha Q+1]^{\delta-1}
$$

Finally, we can obtain long run average profit by subtracting long run average costs from long run average revenue as in Equation (8).

$$
\left(r_{1} p_{1}+r_{2} p_{2}-c_{p}-c_{q}\right) E[Y] \lambda-\left[p_{1} h_{1}+p_{2} h_{2}\right] \frac{\alpha Q^{2}}{2 \alpha Q+2}-\frac{K \lambda}{\alpha Q+1}-\lambda\left[\left(p_{1}+p_{2}\right) E[Y]\right]^{\delta}[\alpha Q+1]^{\delta-1}
$$

First and second derivatives of profit function can be obtained as in Equation (9) and Equation (10) respectively.

$$
\begin{aligned}
& -\left[p_{1} h_{1}+p_{2} h_{2}\right] \frac{\alpha Q(\alpha Q+2)}{2(\alpha Q+1)^{2}}+\frac{\alpha K \lambda}{(\alpha Q+1)^{2}}-\lambda\left[\left(p_{1}+p_{2}\right) E[Y]\right]^{\delta} \alpha(\delta-1)[\alpha Q+1]^{\delta-2} \\
& -\left[p_{1} h_{1}+p_{2} h_{2}\right] \frac{\alpha}{(\alpha Q+1)^{3}}-\frac{2 a^{2} K \lambda}{(\alpha Q+1)^{3}}-\lambda\left[\left(p_{1}+p_{2}\right) E[Y]\right]^{\delta} \alpha^{2}(\delta-1)(\delta-2)[\alpha Q+1]^{\delta-3}
\end{aligned}
$$

In second derivative, third term is always negative since $\delta<1$. First and second terms are also negative. As a result, second derivative is always negative. For this reason, the long run average profit function is concave and optimum $Q$ value can be found by the following equation.

$$
-\left[p_{1} h_{1}+p_{2} h_{2}\right] \frac{\alpha Q(\alpha Q+2)}{2(\alpha Q+1)^{2}}+\frac{\alpha K \lambda}{(\alpha Q+1)^{2}}-\lambda\left[\left(p_{1}+p_{2}\right) E[Y]\right]^{\delta} \alpha(\delta-1)[\alpha Q+1]^{\delta-2}=0
$$

\subsection{Variable Acquisition Fee}

In the previous subsection, we assume that the acquisition fee is fixed. However, sometimes collection centers may want to use the acquisition fee as an incentive factor, e.g., they may offer higher acquisition fees to collect more EOL products in a unit time. In such a case, arrival rate is assumed to be a function of the acquisition fee. In this subsection, we focus on this case and we propose a model which determines optimal acquisition fee and optimal dispatching quantity in a coordinated manner. Let the arrival rate $\lambda\left(c_{p}\right)$, be a function of the acquisition fee, $c_{p}$. In this case, the long run average profit function will be as in Equation (12).

$$
\left(r_{1} p_{1}+r_{2} p_{2}-c_{p}-c_{q}\right) E[Y] \lambda\left(c_{p}\right)-\left[p_{1} h_{1}+p_{2} h_{2}\right] \frac{\alpha Q^{2}}{2 \alpha Q+2}-\frac{K \lambda}{\alpha Q+1}-\lambda\left(c_{p}\right)\left[\left(p_{1}+p_{2}\right) E[Y]\right]^{\delta}[\alpha Q+1]^{\delta-1}
$$

Concavity of this function can be investigated by using the techniques for multivariate functions. However, in order to investigate the concavity of this function, the function, $\lambda\left(c_{p}\right)$ should be defined first. Although it may be possible to obtain the concavity for some $\lambda\left(c_{p}\right)$ functions, this is not possible for all the cases. For this reason,

\begin{tabular}{|c|c|c|c|c|c|c|}
\hline$c_{p}$ & $c_{q}$ & $h_{1}$ & $h_{2}$ & $\mathrm{~K}$ & $\bar{\lambda}$ & $A$ \\
\hline 25 & 20 & 1 & 2 & 250 & 5 & 0.444 \\
\hline$r_{1}$ & $r_{2}$ & $p_{1}$ & $p_{2}$ & $\delta$ & $\mathrm{E}[\mathrm{Y}]$ & $\beta$ \\
\hline 60 & 110 & 0,7 & 0,4 & 0,90 & 2.25 & 0.2 \\
\hline
\end{tabular}
we use direct search algorithms to obtain the optimal values of variables instead of benefiting from the concavity.

\section{Computational Results}

In this part of the study, we present computational results. We assume that $\lambda\left(c_{p}\right)=\beta c_{p}$ where $\beta$ is a real number. Parameters of the model are given below in Table 2.

In the fixed acquisition fee model, we directly use an acquisition fee of 25 as seen in Table 2. In the variable acquisition fee model, the model itself determines the optimal acquisition fee with optimal quantity in a coordinated manner. Computational results are given below in Table 3. 
TABLE III: Computational Results

\begin{tabular}{lccc}
\hline \hline Model & Acquisition Fee & Quantity & Profit \\
\hline Fixed Acquisition Fee & 25 & 60 & 364.543 \\
Variable Acquisition Fee & 31 & 67 & 379.274 \\
\hline \hline
\end{tabular}

It is seen in Table 3 that the model determines the acquisition fee as 31 . Although the acquisition fee is increased from 25 to 31, collection center's average profit is also increased as a result of the increase in arrival rate. From these results, it is possible to claim that variable acquisition fee model works better compared to fixed acquisition fee model. In other words, determining the optimal acquisition fee significantly effects the system profit and thus, it should be used beside the optimal quantity.

For the first model, the model determined the optimal quantity as 60. It means that, dispatch decision will be given if the number of EOL products brought to the collection center exceeds 60 . As it is seen in table, this value is obtained as 67 in the second model as a result of increased arrival rate.

\section{Sensitivity Analysis and Managerial Implications}

In this part of the study, we present some sensitivity analysis in order to see the effects of the parameters on the results. In each case, we only change the value of one parameter and the others are remained as fixed. Computational results for different cases are summarized in Table 4.

TABLE IV: Sensitivity Analysis

\begin{tabular}{cccc}
\hline \hline Changing Parameter & Acquisition Fee & Quantity & Profit \\
\hline Base Case & 25 & 60 & 364.543 \\
$h_{2}=1$ & 25 & 70 & 376.995 \\
$h_{2}=5$ & 25 & 44 & 335.519 \\
$r_{1}=30$ & 25 & 60 & 128.294 \\
$r_{1}=100$ & 25 & 60 & 679.544 \\
$p_{2}=0.2$ & 25 & 70 & 130.815 \\
$p_{2}=0.8$ & 25 & 48 & 836.547 \\
$c_{q}=10$ & 25 & 60 & 477.044 \\
$c_{q}=30$ & 25 & 60 & 252.044 \\
$K=150$ & 25 & 46 & 385.033 \\
$K=400$ & 25 & 76 & 340.400 \\
\hline \hline
\end{tabular}

Following inferences can be made from Table 4. First, we see that value of revenue and quality control cost affect the profit but they do not have any effect on the optimal quantity. For instance, when we increase $r_{1}$ to 100 or decrease it to 30 , optimal quantity does not change and remains as 60 . Secondly, when we decrease holding cost of second component, $h_{2}$, we see that optimal quantity increases and when we increase it, optimal quantity decreases. It means that keeping higher level of inventory is better if the holding cost is low and keeping lower level of inventory is better if the holding cost is high. A similar inference can be made also for fixed transportation cost. If fixed transportation cost is higher, the model decides to keep higher level of inventory and if it is low, the model decides keeping lower level of inventory. Finally, probability of reusability has also an important effect on optimal quantity. When we decrease $p_{2}$ to 0.2 , we see that optimal quantity increases to 70 but when we increase it to 0.8 , optimal quantity decreases to 48 . This is reasonable since we focus on the quantity of EOL products. If the probability of obtaining one of the components decreases, less components are expected to be obtained from a set of EOL products and for this reason, the collection center waits for higher amounts of EOL products to have enough components. However, if that probability is high, higher amount of 
components are expected to be obtained from a set of EOL products. Therefore, the model gives the dispatch decision in lower quantity of EOL products.

\section{Conclusion}

In this study, we propose two renewal-reward process based models for the dispatch decision of collection centers. We assume that the arrival times, batch sizes and quality of components are random. These assumptions make our model more real-life oriented.

Computational results show that variable acquisition fee model performs better compared to fixed acquisition fee model since it optimizes both acquisition fee and quantity in a coordinated manner. For this reason, it can be claimed that determining and using the optimal acquisition fee is essential and important. In addition, we see that the company may obtain higher profit by providing some incentives (e.g. increasing the acquisition fee).

Finally, we see from the sensitivity analysis that all the parameters except the quality control cost and revenue obtained from a component have an effect on both the optimal quantity and profit. However, quality control cost and revenue obtained from a component do not have a significant effect on optimal quantity, although they affect the profit.

\section{References}

[1] V. Guide Jr., and L. Wassenhove, “The Evolution of Closed-Loop Supply Chain Research”, Operations Research, vol. 57, pp. 10-18, 2009

https://doi.org/10.1287/opre.1080.0628.

[2] M. Pishvaee, M. Rabbani, and S. Torabi, "A robust optimization approach to closed-loop supply chain network design under uncertainty”, Applied Mathematical Modelling, vol. 35, pp. 637-649, 2011

https://doi.org/10.1016/j.apm.2010.07.013.

[3] K. Govindan, H. Soleimani, and D. Kannan, "Reverse logistics and closed-loop supply chain: A comprehensive review to explore the future", European Journal of Operational Research, vol. 240, pp. 603-626, 2015.

https://doi.org/10.1016/j.ejor.2014.07.012

[4] X. Hong, Z. Wang, D. Wang, and H. Zhang, "Decision models of closed-loop supply chain with remanufacturing under hybrid dual-channel collection”, Int. J. of Advances Manufacturing Technology, vol. 68, pp. 1851-1865, 2013. ttps://doi.org/10.1007/s00170-013-4982-1

[5] C. Chuang, C. Wang, and Y. Zhao, "Closed-loop supply chain models for a high-tech product under alternative reverse channel and collection cost structures", International Journal of Production Economics, vol. 156, pp. 108-123, 2014. https://doi.org/10.1016/j.jpe.2014.05.008

[6] Y. Shi, J. Nie, T. Qu L. Chu, and D. Sculli, "Choosing reverse channels under collection responsibility sharing in a closed-loop supply chain with re-manufacturing”, Journal of Intelligent Manufacturing, vol. 26, pp. 387-402, 2015. https://doi.org/10.1007/s10845-013-0797-z

[7] I. Hong, and J. Yeh, "Modeling closed-loop supply chains in the electronics industry: A retailer collection application", Transportation Research Part E, vol. 48, pp. 817-829, 2012 https://doi.org/10.1016/j.tre.2012.01.006.

[8] G. Tagaras, and C. Zikopoulos, "Optimal location and value of timely sorting of used items in a remanufacturing supply chain with multiple collection sites", Int. Journal of Production Economics, vol. 115, pp. 424-432, 2008.

ttps://doi.org/10.1016/j.ijpe.2007.10.026

[9] Q. Gu, and G. Tagaras, "Optimal collection and remanufacturing decisions in reverse supply chains with collector's imperfect sorting”, International Journal of Production Research, vol. 52, pp. 5155-5170, 2014. 
https://doi.org/10.1080/00207543.2014.899720

[10] C. Zikopoulosa, and G. Tagaras, "Reverse supply chains: Effects of collection network and returns classification on profitability”, European Journal of Operational Research, vol. 246, pp. 435-449, 2015.

https://doi.org/10.1016/j.ejor.2015.04.051

[11] N. Zaarour, E. Melachrinoudis, M. Solomon, and H. Min, "The optimal determination of the collection period for returned products in the sustainable supply chain”, Int. J. of Logistics Res. and Applications, vol. 17, pp. 35-45, 2014. https://doi.org/10.1080/13675567.2013.836160

[12] T. Watanabe, and E. Kusukawa, "Optimal operation for green supply chain considering demand information, collection incentive and quality of recycling parts" Industrial Eng. \& Management Systems, vol. 13, pp. 129-147, 2014. https://doi.org/10.7232/iems.2014.13.2.129

[13] S. Cetinkaya, and J. Bookbinder, "Stochastic models for the dispatch of consolidated shipments" Transportation Research Part B, vol. 37, pp. 747-768, 2003.

https://doi.org/10.1016/S0191-2615(02)00060-7 